\title{
Effective Bandwidth of Multimedia Traffic in Packet Wireless CDMA Networks with LMMSE Receivers: A Cross-Layer Perspective
}

\author{
Fei Yu and Vikram Krishnamurthy
}

\begin{abstract}
We propose a novel concept of cross-layer effective bandwidth that characterizes the unified resource usage taking into account both physical layer linear minimum mean square error (LMMSE) receivers and statistical characteristics of the packet traffic in code division multiple access (CDMA) networks. Based on the concept of cross-layer effective bandwidth, we develop an optimal connection admission control (CAC) scheme for variable bit rate packet traffic with QoS constraints at both physical and network layers. By introducing a small signal-tointerference ratio (SIR) outage probability using the concept of cross-layer effective bandwidth, the capacity of CDMA networks in the proposed CAC scheme can be increased significantly compared to some existing schemes. The effectiveness of the proposed approaches is demonstrated by numerical examples.
\end{abstract}

Index Terms-Code division multiple access, effective bandwidth, cross-layer design, admission control.

\section{INTRODUCTION}

$\mathbf{I}$ $\mathrm{N}$ RECENT years, there have been significant efforts to develop sophisticated code division multiple access (CDMA) physical layer communication techniques, e.g., multiuser receivers that mitigate not only the background noise but also the interference from other users of the multiaccess channel. In particular, the linear minimum-mean square error (LMMSE) receiver [1], [2] has received considerable attention. A notion of effective bandwidth is proposed in [1] to characterize the resource needed for a connection to meet the signal-tointerference ratio (SIR) requirement.

Although much work has been done in the analysis of CDMA networks, most of previous work concentrates on physical layer and does not consider the stochastically varying traffic load. Consequently, the obtained results may only applicable to a "worst case" scenario with constant bit rate traffic. However, future wireless systems are expected to support variable bit rate packet traffic. In order to properly analyze a CDMA system, it is important to consider the characteristics of the carried traffic at both physical and network levels.

The concept of effective bandwidth of a traffic source at network level has been well developed [3], where the physical layer is assumed to be a wireline network. The network layer effective bandwidth may not be applicable to CDMA networks with LMMSE receivers as wireless fading channels are not considered in [3].

Manuscript received April 15, 2004; revised September 9, 2004; accepted January 14,2005 . The associate editor coordinating the review of this letter and approving it for publication was R. Fantacci. This work is supported by the Natural Science and Engineering Research Council of Canada.

The authors are with the Department of Electrical and Computer Engineering, University of British Columbia, 2356 Main Mall, Vancouver, BC, Canada V6T 1 Z4 (e-mail: \{feiy, vikramk\}@ece.ubc.ca).

Digital Object Identifier 10.1109/TWC.2006.03009.
Since the concept of effective bandwidth is useful at both physical and network levels, why not consider them jointly? In this letter, we propose a novel notion of cross-layer effective bandwidth, by which we can have a unified measure of resource usage taking into account the QoS requirements at both physical and network levels. The contributions of this work are two-fold:

1) The concept of cross-layer effective bandwidth is new. Unlike the previous work [1], [3] that considers only one layer (e.g., only physical layer or only network layer), our approach considers both LMMSE physical layer and statistical characteristics of the packet traffic at network layer.

2) Based on the concept of cross-layer effective bandwidth, we propose an optimal connection admission control (CAC) scheme for variable bit rate packet traffic with QoS constraints at both physical and network layers. Instead of guaranteeing the SIR at all time instants as in [4], [5], we guarantee the SIR outage probability. With a small SIR outage probability, the proposed CAC scheme can dramatically improve the network utilization. Unlike the CAC schemes based on Markov modulated Poisson process (MMPP) traffic models in [6], a variety of traffic models can be used in the proposed CAC scheme with the help of cross-layer effective bandwidth.

Authors in [7] propose to use the effective bandwidth concept in CDMA networks. However, the evaluation of CDMA capacity in [7] is heuristic as there is no mention of the receiver structure. Therefore, the effective bandwidth concept in [7] may only characterize the stochastically varying traffic but no fading channels. The predictive temporal structure of the multi-access interference (MAI) is investigated in [8] taking into account both channel fading and traffic burstiness. However, effective bandwidth is not considered in [8].

In numerical examples, we show that the admissible region obtained from the cross-layer effective bandwidth approach has good approximation to the simulations. In addition, the capacity of CDMA networks can be increased significantly in the proposed CAC scheme compared to the schemes in [4], [5], [7].

The rest of the letter is organized as follows. Section 2 describes the CDMA physical layer and network layer effective bandwidth. Section 3 presents the definition of crosslayer effective bandwidth. Section 4 presents the optimal CAC scheme. Some numerical examples are given in Section 5. Finally, we conclude this study in Section 6. 


\section{MODEL DESCRIPTION}

\section{A. Fading Channel and Linear Multiuser Detector Physical Layer Model}

As in [1], [4], [5], we consider a synchronous CDMA system with spreading gain $N$ and $K$ users. ${ }^{1}$ The signature sequences of all users are independent and randomly chosen. Due to multi-path fading, each user appears as $L$ resolvable paths at the receiver. The path $l$ of user $k$ is characterized by its estimated average channel gain $\bar{h}_{k l}$ and its estimation error variance $\xi_{k}^{2}$. The channel model is conditioned on the slow fading (free space path loss and shadow fading). The attenuated transmitted power from user $k$ is $P_{k}=Z_{k} P_{k}^{t}, k=1,2, \ldots, K$, where $Z_{k}$ is the path loss and $P_{k}^{t}$ is the transmitted power. Linear minimum mean square error (LMMSE) detectors are used at the receiver. In a large system (both $N$ and $K$ are large) with background noise $\sigma^{2}$, the SIR for the LMMSE receiver of a user (say, the first one) can be expressed approximately as [2] $\operatorname{SIR}_{1}=\left(P_{1} \sum_{l=1}^{L}\left|\bar{h}_{1 l}\right|^{2} \eta\right) /\left(1+P_{1} \xi_{1}^{2} \eta\right)$, where $\eta$ is the unique fixed point in $(0, \infty)$ that satisfies $\eta=\left[\sigma^{2}+\right.$ $\left.(1 / N) \sum_{k=2}^{K}\left((L-1) I\left(\xi_{k}^{2}, \eta\right)+I\left(\sum_{l=1}^{L}\left|\bar{h}_{k l}\right|^{2}+\xi_{k}^{2}, \eta\right)\right)\right]^{-1}$ and $I(\nu, \eta)=\nu /(1+\nu \eta)$. Assume that there are $J$ classes of traffic in the system. An important physical layer performance measure of class $j$ users is $\operatorname{SIR}_{j}$, which should be kept above the target value $\omega_{j}$. In [5], it is shown that a minimum received power solution exists such that all users in the system meet their target SIRs if and only if $\omega_{j}<\left|\bar{h}_{j}^{i}\right|^{2} / \xi_{j}^{i}$ and

$$
\sum_{j=1}^{J} \sum_{i=1}^{n_{j}} R_{j}^{i} \frac{\Upsilon_{j}^{i}}{N}<1
$$

where $\left|\bar{h}_{j}^{i}\right|^{2}$ is the average channel gain of the $i$ th class $j$ user, $\left|\bar{h}_{j}^{i}\right|^{2}=\sum_{l=1}^{L}\left|\bar{h}_{j l}^{i}\right|^{2}, n_{j}$ is the number of class $j$ users, $R_{j}^{i}$ is the number of signature sequences assigned to the $i$ th user of class $j$ to make it transmit at $R_{j}^{i}$ times the basic rate (obtained using the lowest spreading gain $N$ ) and

$$
\Upsilon_{j}^{i}=(L-1) \omega_{j} \frac{\xi_{j}^{i^{2}}}{\left|\bar{h}_{j}^{i}\right|^{2}}+\frac{\omega_{j}\left(1+\frac{\xi_{j}^{i 2}}{\left|h_{j}^{i}\right|^{2}}\right)}{1+\omega_{j}} .
$$

The minimum received power solution for the first class 1 user is [5] $P_{1}=\omega_{1} \sigma^{2} /\left|\bar{h}_{1}^{1}\right|^{2}\left(1-\omega_{1} \xi_{1}^{1} /\left|\bar{h}_{1}^{1}\right|^{2}\right)(1-$ $\left.(1 / N) \sum_{j=1}^{J} \sum_{i=1}^{n_{j}} R_{j}^{i} \Upsilon_{j}^{i}\right)$. The capacity of the system is restricted by the power control feasibility condition (1). The SIR outage probability in CDMA networks with LMMSE receivers can be expressed as

$$
P^{\text {out }}=P\left\{\sum_{j=1}^{J} \sum_{i=1}^{n_{j}} R_{j}^{i} \frac{\Upsilon_{j}^{i}}{N} \geq 1\right\}
$$

\section{B. Network Layer Effective Bandwidth}

Consider a bufferless communication multiplexer with a single output. There are $J$ classes of input traffic with $n_{j}$

\footnotetext{
${ }^{1}$ The proposed cross-layer effective bandwidth concept is also applicable to an asynchronous CDMA system. In [9], it is shown that an asynchronous CDMA system with $K$ users can be viewed as a synchronous CDMA system with $M K$ users, where $M$ is the frame length. For simplicity of the presentation, we only consider synchronous systems in this letter.
}

connections of class $j$ traffic. The aggregate input traffic is $Y=\sum_{j=1}^{J} \sum_{i=1}^{n_{j}} Y_{j}^{i}$, where $Y_{j}^{i}$ is the $i$ th class $j$ traffic. Assume that the output capacity is $C$. The congestion probability of this system is

$$
P^{\text {cong }}=P\left\{\sum_{j=1}^{J} \sum_{i=1}^{n_{j}} Y_{j}^{i} \geq C\right\} .
$$

Given the statistical characteristics of traffic sources and their congestion probability requirements, the actual bandwidth that a connection requires lies between its mean rate and its peak rate. This bandwidth is generally referred to as the effective bandwidth of the traffic source. Assume that $Y_{j}[0, t]$ is the amount of work that arrives from a class $j$ source in the time interval $[0, t]$, and $Y_{j}[0, t]$ has stationary increments. The definition of network layer effective bandwidth of class $j$ traffic is [3]

$$
\alpha_{j}(s, t)=\frac{1}{s t} \log \mathbf{E}\left[e^{s Y_{j}[0, t]}\right], s, t \in \mathbb{R}_{+},
$$

where $\mathbf{E}$ is the expectation, $s$ and $t$ are system parameters defined by the characteristics of the source, its QoS requirements, and the link capacity. The time parameter $t$ (measured in, e.g., milliseconds) indicates the time scales that are important for buffer overflow in buffer models: A small value of $t$ indicates that fast time scales are responsible for buffer overflow, whereas a large value of $t$ indicates that slow time scales are responsible for buffer overflow. In bufferless models, the instantaneous arrival rates of individual traffic sources contribute to packet loss, corresponding to the choice $\alpha_{j}(s)=\lim _{t \rightarrow 0} \alpha_{j}(s / t, t)$. The space parameter $s$ (measured in, e.g., bits ${ }^{-1}$ ) indicates the degree of statistical multiplexing and depends on the size of the peak rates of the multiplexed sources relative to the link capacity. Particularly, for the link capacity much larger than the peak rates of the multiplexed sources, $s$ tends to zero and $\alpha_{j}(s, t)$ approaches the mean rate of the source, corresponding to a large degree of statistical multiplexing, while for the link capacity not much larger than the peak rates of the sources, $s$ is large and $\alpha_{j}(s, t)$ approaches the peak rate of the source, corresponding to a low degree of statistical multiplexing.

\section{CROSS-LAYER EFFECTIVE BANDWIDTH}

\section{A. Definition of Cross-Layer Effective Bandwidth}

Comparing (3) with (4), we can see the similarity between CDMA networks with LMMSE receivers and wireline networks. From a mathematical point of view, there is a scalar $\Upsilon_{j}^{i} / N$ in (3). It is very interesting to observe that the scalor $\Upsilon_{j}^{i} / N$ contains all the information about the physical layer LMMSE receivers. Since the definition of network layer effective bandwidth (5) is useful in deriving the congestion probability (4), we can develop a concept of cross-layer effective bandwidth to derive the SIR outage probability (3). This motivates us to define the cross-layer effective bandwidth of a traffic source as follows:

Definition 3.1: Let $L$ denote the number of resolvable paths that each user appears at the LMMSE receiver, $\left|\bar{h}_{j}^{i}\right|^{2}$ denote the estimated average channel gain and $\xi_{j}^{i^{2}}$ denote the estimation error variance of the $i$ th class $j$ connections with a SIR target 
value $\omega_{j}$ in a CDMA system with spreading gain $N$. Let $R_{j}^{i}[0, t]$ denote the amount of work generated from the $i$ th connection of class $j$ in the time interval $[0, t]$, and $R_{j}^{i}[0, t]$ is assumed to have stationary increments. The cross-layer effective bandwidth of this connection is

where $\Upsilon_{j}^{i}$ is defined in (2).

Remark: The definition above incorporates both physical layer LMMSE receivers and network layer packet traffic information. Specifically, $R_{j}^{i}[0, t]$ contains the statistical characteristics of the packet traffic, whereas the information about the physical layer LMMSE receivers is abstracted by $\Upsilon_{j}^{i} / N$ in (6). $L, N, h, \xi, \omega$ are systems parameters defined by physical layer LMMSE receivers. System parameters $s, t$ are defined similarly as those of network layer effective bandwidth (5). The physical interpretation of $\alpha_{j}(L, N, h, \xi, \omega, s, t)$ is that using the cross-layer effective bandwidth concept we can have a unified measure of resource usage given the physical layer wireless fading channels in CDMA systems with LMMSE receivers, statistical characteristics of the packet traffic and QoS requirements at both physical and network layers.

\section{B. Derivation of SIR Outage Probability Using Cross-Layer Effective Bandwidth}

We use SIR outage probability as a QoS measure in wireless packet CDMA networks. Instead of guaranteeing the SIR at all time instants, we guarantee the SIR outage probability. This formulation is motivated by the design of packet wireline networks. It is well known [10] that allocating all connections with their peak rates guarantees no packet loss, but results in the lowest utilization. Therefore, most bandwidth allocation schemes in wireline networks allow a small packet loss probability to increase the network utilization [10]. Similarly, since most applications in wireless networks can tolerate small probability of SIR outage, we can use SIR outage probability as a QoS measure and keep it below a target value $\zeta$. The SIR outage probability can be estimated by the following wellknown Chernoff bound [11] approximation

$$
P^{\text {out }}=P\left\{\sum_{j=1}^{J} \sum_{i=1}^{n_{j}} R_{j}^{i} \frac{\Upsilon_{j}^{i}}{N} \geq 1\right\} \approx e^{\Lambda(1)},
$$

where $\Lambda(v)=\inf _{s}[s \alpha-s v], \alpha=\sum_{j=1}^{J} \sum_{i=1}^{n_{j}} \alpha_{j}^{i}$, and $\alpha_{j}^{i}$ is the scaled logarithmic moment generating function of the instantaneous work load of the $i$ th class $j$ connection in a packet bufferless CDMA system. $\alpha_{j}^{i}=$ $\lim _{t \rightarrow 0} \alpha_{j}^{i}(L, h, \xi, \omega, s / t, t)=(1 / s) \log \mathbf{E}\left[e^{s R_{j}^{i} \Upsilon_{j}^{i} / N}\right]$. The constraint $P^{\text {out }} \leq \zeta$ will be satisfied if the vector $x=$ $\left(n_{1}, n_{2}, \ldots, n_{J}\right)$ lies within the admissible set

$X=\left\{x \in \mathbb{Z}_{+}^{J}: \exp \left\{\inf _{s}\left[s\left(\sum_{j=1}^{J} \sum_{i=1}^{n_{j}} \alpha_{j}^{i}-1\right)\right]\right\} \leq \zeta\right\}$.

The Chernoff bound (7) can be further refined [12] by adding a prefactor. $P^{\text {out }} \approx e^{\Lambda(1)} / s^{*} \sqrt{2 \pi \partial^{2}\left(s^{*} \alpha\right) / \partial s^{2}}$, where $s^{*}$ attains the infimum in (8). The admissible set using the improved bound becomes

\section{Optimal Connection Admission Control in PACKET CDMA NETWORKS}

The interplay between physical layer and network layer plays an important role in designing CDMA networks. Two recent papers [4], [5] study the cross-layer admission control problem. However, only constant bit rate traffic is considered in [4], [5]. Using the concept of cross-layer effective bandwidth proposed in this letter, we can design an optimal crosslayer CAC scheme with variable bit rate packet multimedia traffic. We use SIR outage probability as a QoS measure, which is not considered in [4], [5]. Note that, as in [5], the CAC scheme proposed in this letter is also a genuine crosslayer design in which information is exchanged between physical layer and network layer in both directions. We identify the state space, decision epochs, actions, state dynamics, cost and constraints as follows.

\section{A. State Space, Decision Epochs and Actions}

The state space $X$ of the system comprises of any state vector $x=\left[n_{1}, n_{2}, \ldots, n_{J}\right] \in \mathbb{Z}_{+}^{J}$ such that the SIR outage probability constraint can be met. Therefore, the state space of the SMDP can be defined in (8) or (9). We choose the decision epochs to be the set of all connection arrival and departure instances. Action $a(t)$ at decision epoch $t$ is defined as $a(t)=$ $\left[a_{1}(t), a_{2}(t), \ldots, a_{J}(t)\right]$, where $a_{j}(t)$ denotes the action for class $j$ connections. If $a_{j}(t)=1$, admit a class $j$ connection. If $a_{j}(t)=0$, the connection is rejected. The action space can be defined as $A=\left\{a: a \in\{0,1\}^{J}, j=1,2, \ldots, J\right\}$. For a given state $x \in X$, a selected action should not result in a transition to a state that is not in $X$. The action space of a given state $x \in X$ is defined as $A_{x}=\left\{a \in A: a_{j}=0\right.$ if $(n+$ $\left.e_{j}^{u}\right) \notin X, j=1,2, \ldots, J$ and $a \neq(0,0, \ldots, 0)$ if $x=$ $(0,0, \ldots, 0)\}$. where $e_{j}^{u} \in\{0,1\}^{J}$ denotes a row vector containing only zeros except for the $j$ th component, which is $1, x+e_{j}^{u}$ corresponds to an increase of the number of class $j$ users by 1 .

\section{B. State Dynamics}

Assume that the class $j$ connection arrival process is Poisson with rate $\lambda_{j}$ and the service duration is exponentially distributed with mean $1 / \mu_{j}$. The state dynamics can be characterized by the state transition probabilities of the embedded chain $p_{x y}(a)$ and the expected sojourn time $\tau_{x}(a)$ for each state-action pair. $\tau_{x}(a)=\left[\sum_{j=1}^{J} \lambda_{j} a_{j}+\sum_{j=1}^{J} \mu_{j} n_{j}\right]^{-1}$. The state transition probabilities of the embedded Markov chain are

$$
p_{x y}(a)=\left\{\begin{array}{lll}
\lambda_{j} a_{j} \tau_{x}(a), & \text { if } y=x+e_{j}^{u}, \quad j=1,2, \ldots, J \\
\mu_{j} n_{j} \tau_{x}(a), & \text { if } y=x-e_{j}^{u}, \quad j=1,2, \ldots, J \\
0, & \text { otherwise. }
\end{array}\right.
$$

\section{Performance Criterion, Cost Function, and Constraints}

The average cost is considered as the performance criterion in this letter. Based on the action $a$ taken in a state $x$, a cost $c(x, a)$ occurs to the network. Authors in [4] show that the blocking probability can be expressed as an average cost 


$$
\begin{gathered}
\alpha_{j}^{i}(L, N, h, \xi, \omega, s, t)=\frac{1}{s t} \log \mathbf{E}\left[e^{s R_{j}^{i}[0, t] \Upsilon_{j}^{i} / N}\right], L, N \in \mathbb{Z}_{+}, h, \xi, \omega, s, t \in \mathbb{R}_{+}, \\
X=\left\{x \in \mathbb{Z}_{+}^{J}: \frac{1}{s^{*} \sqrt{2 \pi \frac{\partial^{2}}{\partial s^{2}}\left(s^{*} \sum_{j=1}^{J} \sum_{i=1}^{n_{j}} \alpha_{j}^{i}\right)}} \exp \left[s^{*}\left(\sum_{j=1}^{J} n_{j} \alpha_{j}-1\right)\right] \leq \zeta\right\} .
\end{gathered}
$$

TABLE I

PARAMETERS USED IN NUMERICAL EXAMPLES.

\begin{tabular}{c|c|c}
\hline Parameter & Notation & Value \\
\hline \hline system bandwidth & $W$ & $5 \mathrm{MHz}$ \\
\hline spreading gain & $N$ & 512 \\
\hline target SIR for voice traffic & $\omega_{1}$ & $7 \mathrm{~dB}$ \\
\hline estimated average channel gain for voice traffic & $\left|h_{1}\right|^{2}$ & 1 \\
\hline channel estimation error variance for voice traffic & $\xi_{1}^{2}$ & 0.02 \\
\hline data transmission rate in state ON for voice traffic & $R_{1}$ & $15 \mathrm{kbps}$ \\
\hline rate from ON to OFF for voice traffic & $\alpha_{1}$ & 0.4 \\
\hline rate from OFF to ON for voice traffic & $\beta_{1}$ & 0.4 \\
\hline service rate for voice traffic & $\mu_{1}$ & 0.005 \\
\hline target SIR for video traffic & $\omega_{2}$ & $10 \mathrm{~dB}$ \\
\hline estimated average channel gain for video traffic & $\left|h_{2}\right|^{2}$ & 1 \\
\hline channel estimation error variance for video traffic & $\xi_{2}^{2}$ & 0.05 \\
\hline data transmission rate in state NORMAL for video traffic & $R_{21}$ & $30 \mathrm{kbps}$ \\
\hline data transmission rate in state BURST for video traffic & $R_{22}$ & $60 \mathrm{kbps}$ \\
\hline rate from state NORMAL to state BURST for video traffic & $\alpha_{2}$ & 0.024 \\
\hline rate from state BURST to state NORMAL for video traffic & $\beta_{2}$ & 0.076 \\
\hline service rate for video traffic & $\mu_{2}$ & 0.004 \\
\hline
\end{tabular}

criterion in the CAC setting. Following [4], we define the cost as $c(x, a)=\sum_{j=1}^{J} w_{j}\left(1-a_{j}\right)$, where $w_{j} \in \mathbb{R}_{+}$is the weight associated with class $j$.

In the formulated problem, the SIR outage probability constraint in the system can be guaranteed by restricting the state space in (8) and (9). In addition, the blocking probability for class $j$ can be defined as the fraction of time the system is in a set of states $X_{j}^{b} \subset X$ and the chosen action is in a set of actions $A_{x_{j}^{b}} \subset A$, where $x_{j}^{b} \in X_{j}^{b}$ and $A_{x_{j}^{b}}=\{a \in A$ : $\left.a_{j}=0\right\}, P_{j}^{b}=\sum_{x \in X_{j}^{b}} \sum_{a \in A_{x_{j}^{b}}} \tau_{x}(a) / \sum_{x \in X} \sum_{a \in A} \tau_{x}(a)$. The blocking probability constraints can be expressed as $P_{j}^{b} \leq \gamma_{j}, j=1,2, \ldots, J$, which can be addressed in the linear programming formulation by defining a cost function related to these constraints $c_{j}^{b}(x, a)=\left(1-a_{j}\right), j=1,2, \ldots, J$.

\section{Linear Programming Solution to the SMDP}

The optimal policy $u^{*}$ of the SMDP is obtained by solving the following linear program [13].

$$
\min _{z_{x a} \geq 0, x \in X, a \in A_{x}} \sum_{x \in X} \sum_{a \in A_{x}} \sum_{j=1}^{J} w_{j}\left(1-a_{j}\right) \tau_{x}(a) z_{x a}
$$

subject to

$$
\begin{aligned}
\sum_{a \in A_{y}} z_{y a}-\sum_{x \in X} \sum_{a \in A_{x}} p_{x y}(a) z_{x a} & =0, y \in X \\
\sum_{x \in X} \sum_{a \in A_{x}} z_{x a} \tau_{x}(a) & =1, \\
\sum_{x \in X} \sum_{a \in A_{x}}\left(1-a_{j}\right) z_{x a} \tau_{x}(a) & \leq \gamma_{j}, j=1,2, \ldots, J .
\end{aligned}
$$

\section{NumericAl EXAMPLES}

The numerical values used for the system parameters in the numerical examples are given in Table 1 . We consider two classes of traffic, voice and MPEG video. The voice traffic is modeled as an ON/OFF process. The rate in state ON is 15 kbps corresponding to an equivalent spreading gain $N=256$. A Markov model with two states, NORMAL and BURST, is used for the MPEG traffic. The rate in state NORMAL is 30 kbps corresponding to an equivalent spreading gain $N=128$ and the rate in state BURST is two times of that in state NORMAL.

We show the performance of cross-layer effective bandwidth by comparing the admissible regions calculated from (8) and (9) with that from the simulations. In the simulations, we keep adding more connections to the system until the SIR outage probability constraint, 0.005 , is violated. Fig. 1 shows the results. We can see that the admissible regions obtained from the effective bandwidth approaches using both Chernoff bound and improved bound are smaller than that from the simulations, which means that the approaches using crosslayer effective bandwidth are conservative compared to the 


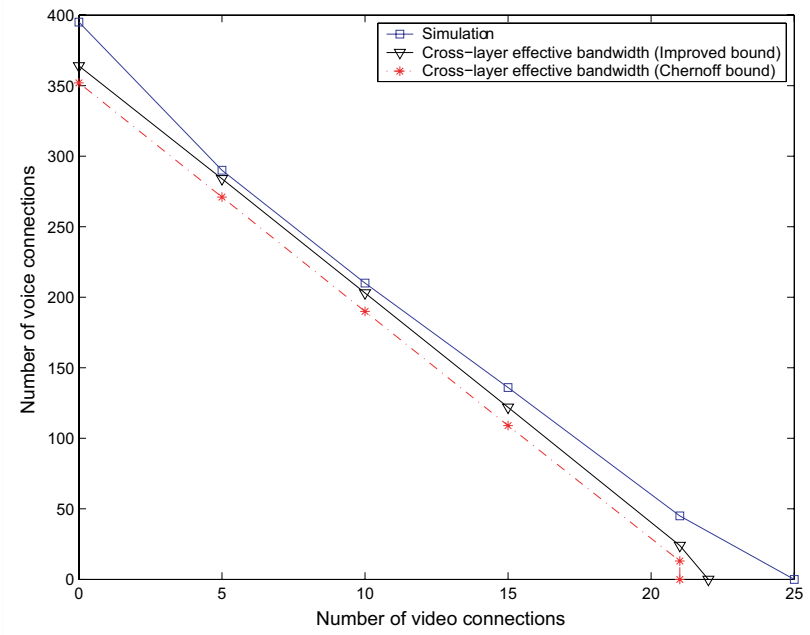

Fig. 1. Admissible regions obtained from cross-layer effective bandwidth and simulations.

simulations, especially when Chernoff bound is used. However, we observe that the admissible region using improved bound can have good approximation to the simulations. This shows the effectiveness of the concept of cross-layer effective bandwidth. We use improved bound in the following examples.

We compare the admissible regions obtained from crosslayer effective bandwidth to those from peak rate, mean rate and network layer effective bandwidth allocation schemes. Since SIR outage is not allowed in [4], [5], all connections should be allocated with their peak rates to guarantee the SIR at all time instants. Therefore, [4] and [5] are examples of the peak rate scheme. In the mean rate allocation scheme, each connection is allocated with its mean rate. In the network layer effective bandwidth approach [7], only the statistical characteristics of the traffic is considered and LMMSE receivers are not used. Fig. 2 shows the comparisons with peak rate and mean rate schemes. It is observed that the admissible region obtained from the peak rate scheme is much smaller than that from cross-layer effective bandwidth scheme, which means significantly lower network utilization in the peak rate scheme. The mean rate approach can increase the network utilization, but physical layer SIR outage probability cannot be guaranteed. By introducing a small SIR outage probability, 0.005 , the proposed cross-layer effective bandwidth approach can increase network utilization substantially.

Fig. 3 shows the admissible regions obtained from crosslayer effective bandwidth and network layer effective bandwidth approaches. Two values of the number of resolvable paths are used in this example, $L=1$ and $L=5$. We can see that the admissible region obtained from the network layer approach is much smaller that those from cross-layer approaches. This illustrates the importance of considering the physical layer in designing CDMA networks.

\section{Conclusions}

We have presented a novel concept of cross-layer effective bandwidth for variable bit rate multimedia traffic in packet CDMA networks taking into account both physical layer linear minimum mean square error (LMMSE) receivers and

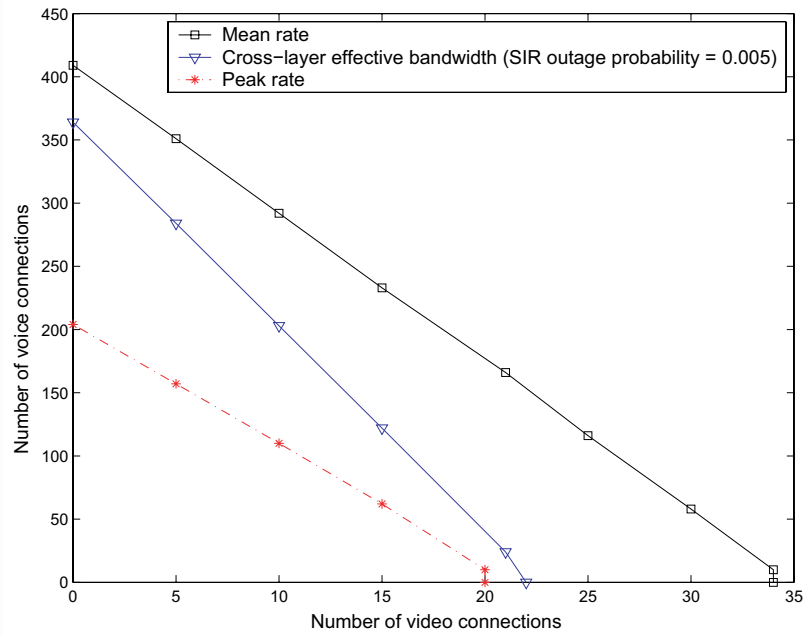

Fig. 2. Admissible regions obtained from mean rate, cross-layer effective bandwidth and peak rate schemes.

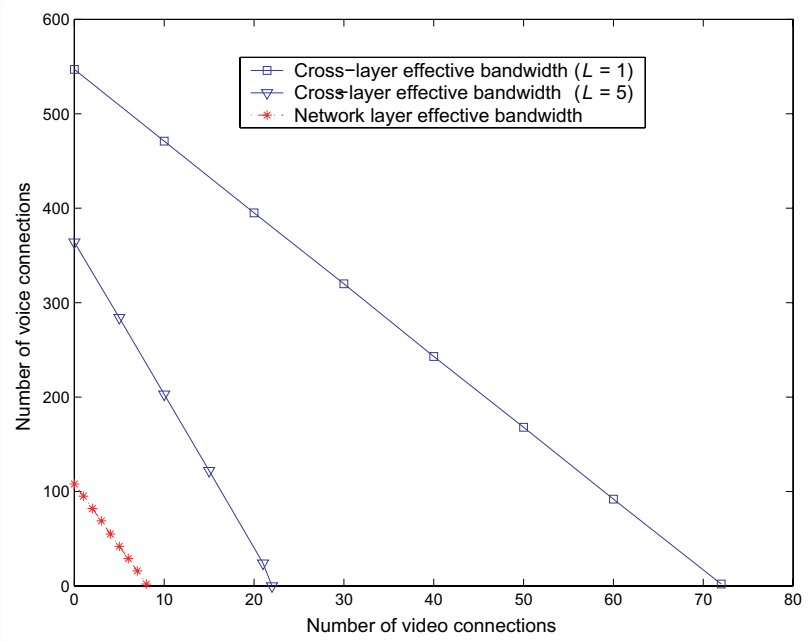

Fig. 3. Admissible regions obtained from cross-layer effective bandwidth and network layer effective bandwidth schemes.

statistical characteristics of the packet traffic. The proposed concept can be a useful tool in the design, analysis and control of packet CDMA networks. An optimal connection admission control scheme was developed based on the concept of cross-layer effective bandwidth. It was shown in numerical results that the admissible region obtained from the crosslayer effective bandwidth can have good approximation to the simulations. We also observed a very significant gain in the capacity when a small SIR outage probability is introduced in packet CDMA networks with LMMSE receivers. Further study is in progress to apply the concept of cross-layer effective bandwidth to other applications, such as tariffs and tele-traffic analysis.

\section{REFERENCES}

[1] D. N. C. Tse and S. Hanly, "Linear multiuser receivers: effective interference, effective bandwith and user capacity," IEEE Trans. Inform. Theory, vol. 45, pp. 641-657, Mar. 1999.

[2] J. Evans and D. N. C. Tse, "Large system performance of linear multiuser receivers in multipath fading channels," IEEE Trans. Inform. Theory, vol. 46, pp. 2059-2078, Sept. 2000. 
[3] F. Kelly, "Notes on effective bandwidths," in Stochastic Networks: Theory and Applications (F. Kelly, S. Zachary, and I. Ziedins, eds.), pp. 141-168, Oxford Press, 1996.

[4] S. Singh, V. Krishnamurthy, and H. V. Poor, "Integrated voice/data call admission control for wireless DS-CDMA systems with fading," IEEE Trans. Signal Proc., vol. 50, pp. 1483-1495, June 2002.

[5] C. Comaniciu and H. V. Poor, "Jointly optimal power and admission control for delay sensitive traffic in CDMA networks with LMMSE receivers," IEEE Trans. Signal Proc., vol. 51, pp. 2031-2042, Aug. 2003.

[6] F. Yu, V. Krishnamurthy and V. C. M. Leung, "Cross-layer optimal connection admission control for variable bit rate multimedia traffic in packet wireless CDMA networks," IEEE Trans. Signal Proc., to appear, 2006.

[7] J. S. Evans and D. Everit, "Effective bandwidth-based admission control for mutliservice CDMA cellular networks," IEEE Trans. Veh. Technol., vol. 48, pp. 36-46, Jan. 1999.
[8] J. Zhang, "Bursty traffic meets fading: a cross-layer design perspective," in Proc. of the 40th Allerton Conf. Commun., Control, and Computing, Oct. 2002.

[9] S. Verdu, "Multiuser detection," in Advances in Statistical Signal Processing (H. Poor and J. Thomas, eds.). Greenwich, CT.: JAI Press, 1993.

[10] M. Schwartz, Broadband Integrated Networks. Prentice Hall, 1996.

[11] H. Chernoff, "A measure of asymptotic efficiency for tests of a hypothesis based on the sum of observation," Ann. Math. Statist., vol. 23, pp. 493-507, 1952.

[12] R. Bahadur and R. Rao, "On deviations of the sample mean," Ann. Math. Statist., vol. 31, pp. 1051-1027, 1960.

[13] H. Tijms, Stochastic Modeling and Analysis: a Computational Approach. New York: Wiley, 1986. 